\title{
Association Between Unmet Essential Social Needs and Influenza Vaccination in US Adults
}

\author{
Daniel J. Parente, MD, PhD (0, Megan J. Murray, MHSA, and Jennifer Woodward, MD, MPH
}

Department of Family Medicine and Community Health, University of Kansas Medical Center, Kansas City, KS, USA.

BACKGROUND: Although social factors influence uptake of preventive services, the association between social needs and influenza vaccination has not been comprehensively evaluated for adults seeking primary care in the USA.

OBJECTIVE: To determine the association between unmet social needs and influenza vaccination.

DESIGN: Retrospective, cross-sectional, multivariable logistic regression.

PARTICIPANTS: Persons completing ambulatory visits in a primary care department at a midwestern, urban, multispecialty, academic medical center between July 2017 and July 2019 ( $N=7955$ individuals included).

MAIN MEASURES: Completion of influenza vaccination in the 2018-2019 influenza season (primary outcome) or any year (secondary outcome) against 11 essential social needs (childcare, companionship, food security, health literacy, home safety, neighborhood safety, housing, health care provider costs, prescription costs, transportation, and utilities). Demographics, diabetic status, COPD, smoking status, office visit frequency, and hierarchical condition category risk scores were included as covariates.

KEY RESULTS: Individuals with transportation vulnerability were less likely to be vaccinated against influenza (current-year aOR 0.65, 95\% CI: 0.53-0.78, $p<0.001$; any-year aOR 0.58, 95\% CI: 0.47-0.71, $p<0.001)$. Poor health literacy promoted any-year, but not current-year, influenza vaccination (any-year aOR 1.30, 95\% CI: $1.01-$ $1.69, p=0.043$ ). Older age, female sex, diabetes, more comorbidities, and more frequent primary care visits were associated with greater influenza vaccination. Persons with Black or other/multiple race and current smokers were less frequently vaccinated.

CONCLUSIONS: Transportation vulnerability, health literacy, smoking, age, sex, race, comorbidity, and office visit frequency are associated with influenza vaccination. Primary care-led interventions should consider these factors when designing outreach interventions.

TRIAL REGISTRATION: Not applicable

KEY WORDS: influenza; social determinants of health; transportation; health literacy; primary care.

Prior presentation None.

Received October 1, 2020

Accepted April 30, 2021

Published online June 15, 2021
J Gen Intern Med 37(1):23-31

DOI: $10.1007 / \mathrm{s} 11606-021-06902-6$

(C) Society of General Internal Medicine 2021

\section{INTRODUCTION}

Widespread utilization of preventive services, including vaccination, is essential for optimizing individual and public health outcomes. Annual influenza vaccination is recommended by the United States Centers for Disease Control and Prevention (CDC) Advisory Committee on Immunization Practices (ACIP) for persons aged 6 months or greater lacking contraindications. ${ }^{1}$ Influenza is a respiratory virus that annually causes $1.5-5.9$ million symptomatic infections and 2-9 thousand excess deaths. ${ }^{2}$ Influenza vaccination prevents an additional 4.8 million symptomatic infections and 7 thousand deaths per year. ${ }^{2}$ Most adults, nevertheless, remain unvaccinated; in the 2018-2019 influenza season, vaccination coverage was only $45.3 \%{ }^{3}$

Sociodemographic factors ${ }^{4}$ and immunization beliefs/attitudes ${ }^{5}$ predict influenza vaccination. ${ }^{4-20}$ Many analyses focus on special populations, including the elderly, ${ }^{4,8-11,13-22}$ children ${ }^{12,23}$ health care workers, ${ }^{6,24}$ persons in Europe, ${ }^{6,9-11,14}$ $16,18,21,25-27$ minority/disadvantaged populations, ${ }^{7,26,28}$ and persons with chronic disease or at high risk of influenzarelated complications. ${ }^{5,10,29}$

Unfulfilled social needs may also influence vaccination uptake. For example, social isolation is associated with decreased rate of influenza immunization among elderly adults. ${ }^{13}$ Moreover, there have been recent calls for multidomain analyses. ${ }^{30}$ In analogy to polygenic approaches-wherein it is understood that many genetic determinants contribute together to health outcomes - multidomain approaches consider that many different social needs domains may also combine to influence health outcomes.

Patients seeking primary care appointments at our institution recently underwent screening for unmet social needs using a multidomain questionnaire (see "METHODS"). ${ }^{31}$ We evaluated whether unmet social needs were associated with influenza vaccination uptake among English-speaking adults seeking primary care.

Primary care physicians are uniquely positioned to improve preventive service uptake, including influenza vaccination. Indeed, personalized physician invitation is associated with increased influenza vaccination. ${ }^{29}$ Patients who use enabling 
services (e.g., care coordination) also have greater access to care, satisfaction, and influenza vaccination. ${ }^{32}$ The most relevant population for primary care-led interventions are individuals empaneled with a primary care physician. We are not aware, however, of any large analyses that have evaluated the impact on influenza vaccination of unfulfilled social needs across numerous domains in this population. We evaluated the relationship between current-year and any-year influenza vaccination uptake and 11 domains of social needs among US, English-speaking adults seeking primary care.

\section{METHODS}

\section{Study Design and Population}

We retrospectively analyzed the relationship between currentyear and any-year influenza vaccination status and social needs using a cross-sectional design. This study was crosssectional because influenza vaccination did not always occur after the assessment of unmet social needs. Included in this analysis were all English-speaking adults that had been seen for an ambulatory office visit in the family medicine department at a midwestern, urban, multispecialty, academic health system at least once within a 24-month period spanning July 2017 and July 2019 and who completed a social needs screening questionnaire.

\section{Essential Social Needs Screening Implementation}

Patients were screened for social needs-during routine clinical care-when attending ambulatory appointments. Screening used a variant of the validated Health Leads questionnaire. ${ }^{31}$ Screening identified risks in 11 domains: childcare, transportation, health literacy, food security, stable housing, utilities, companionship, health care costs, medication affordability, home violence, and neighborhood violence (wording shown in Table 1). This instrument differs from screening recommendations by Health Leads only in (1) the division of exposure to violence into two concepts: home and neighborhood violence, guided by the United States Department of Justice Exposure to Violence screening instrument, ${ }^{33}$ and (2) screening for poor health literacy using a dichotomous (yes/no) rather than a Likert scale, consistent with recommendations to use the same scale for all screening questions. ${ }^{31}$ All patients newly establishing care in a primary care clinic within the health system were screened with a 10question screening (all domains except companionship) beginning in October 2017. Patients "newly" establishing care were those not seen by the primary care clinic within the preceding 36 months. In May 2018, screening was expanded to include all 11 domains and include all patients - new or established - within a primary care clinic. Patients were screened prior to their visit through an
Table 1 Prevalence of Unfulfilled Social Needs

\begin{tabular}{|c|c|c|}
\hline Domain & No. (\%) & Screening question \\
\hline $\begin{array}{l}\text { Childcare } \\
\text { assistance }\end{array}$ & $128(1.6)$ & $\begin{array}{l}\text { Do problems getting child care make } \\
\text { it difficult for you to work or study? }\end{array}$ \\
\hline Companionship & $514(6.5)$ & $\begin{array}{l}\text { Do you often feel that you lack } \\
\text { companionship? }\end{array}$ \\
\hline Food security & $494(6.2)$ & $\begin{array}{l}\text { In the last } 12 \text { months, did you ever } \\
\text { eat less than you should because } \\
\text { there wasn't enough money for } \\
\text { food? }\end{array}$ \\
\hline Health literacy & $457(5.7)$ & $\begin{array}{l}\text { Do you have problems } \\
\text { understanding what is told to you } \\
\text { about your medical conditions? }\end{array}$ \\
\hline Home safety & $339(4.3)$ & $\begin{array}{l}\text { Are you afraid you might be hurt in } \\
\text { your home by someone you know? }\end{array}$ \\
\hline Housing & $54(0.7)$ & $\begin{array}{l}\text { Are you worried that in the next } 2 \\
\text { months, you may not have stable } \\
\text { housing? }\end{array}$ \\
\hline $\begin{array}{l}\text { Neighborhood } \\
\text { safety }\end{array}$ & $361(4.5)$ & $\begin{array}{l}\text { Are you afraid you might be hurt in } \\
\text { your apartment building or } \\
\text { neighborhood? }\end{array}$ \\
\hline Provider costs & $253(3.2)$ & $\begin{array}{l}\text { In the last } 12 \text { months, have you } \\
\text { needed to see a doctor, but could not } \\
\text { because of cost? }\end{array}$ \\
\hline Prescription costs & $100(1.3)$ & $\begin{array}{l}\text { In the last } 12 \text { months, did you skip } \\
\text { medications to save money? }\end{array}$ \\
\hline Transportation & $569(7.2)$ & $\begin{array}{l}\text { In the past } 12 \text { months, have you ever } \\
\text { had to go without health care } \\
\text { because you didn't have a way to } \\
\text { get there? }\end{array}$ \\
\hline Utilities & $276(3.5)$ & $\begin{array}{l}\text { In the last } 12 \text { months, has your } \\
\text { utility company shut off your service } \\
\text { for not paying your bills? }\end{array}$ \\
\hline $\begin{array}{l}\text { Any unfulfilled } \\
\text { need }\end{array}$ & $\begin{array}{l}1626 \\
(20.4)\end{array}$ & \\
\hline
\end{tabular}

*The sum of each individual social need does not equal the number of individuals with any unfulfilled need because some individuals expressed more than one social need

electronic patient portal or on the day of their ambulatory visit using a paper form. Screening was updated yearly. Responses were recorded in the electronic medical record (EMR) and each domain included one question with dichotomous (yes/no) response options. Screening was only offered in English. Persons self-reporting a social need were given written information on addressing that need after their office visit and/or referred to a social worker, according to their preference.

\section{Influenza Vaccination}

Current-year (2018-2019 influenza season) and historical influenza vaccination records are entered in the EMR during routine care. Several possible events would result in updates to this data, including (1) immunization against influenza within a health system clinic, (2) patient self-report of immunization entered in the electronic patient portal, (3) nurse or medical assistant inquiry during the rooming process for any ambulatory visit, or (4) reconciliation of outside vaccination records by a physician during an office visit using interconnected external health records. From these data, the date of most recent vaccination (if any) was inferred to be the latest date present in the health record. Individuals were considered vaccinated (1) for the current-year if they were vaccinated 
between August 2018 and April 2019, and (2) in any year if there was any record of influenza vaccination recorded in the medical record. Data extraction occurred in July 2019, after the conclusion of the 2018-2019 influenza vaccination season.

\section{Demographics, Office Visits, Diabetes, COPD, Comorbidities, and Smoking Status}

Patient demographics are universally recorded (age, race, sex, ethnicity) in the EMR for all patients in the health system during routine clinical care. Age was defined as the age in July 2019. Smoking status (current, former, never) is also recorded during routine clinical care and frequently updated during ambulatory primary care visits. Diagnosis of diabetes or chronic obstructive pulmonary disease (COPD) was inferred to exist if it had been previously documented in the EMR. The number of primary care office visits with (1) a physician or nurse practitioner or (2) any provider (physician, nurse, etc.) over a 1-year period between May 2018 and April 2019 was determined to estimate yearly frequency of clinical contact. Centers for Medicare and Medicaid Services Hierarchical Condition Category (CMS-HCC) risk scores-which model projected health care costs ${ }^{34,35}$ and increase as greater comorbidities are noted - were assigned the earliest available score among two datasets extracted for April or September 2019, and included HCC diagnoses annotated over the preceding 3 years.

\section{Data Extraction}

Demographics (age, race, sex, ethnicity), health information (smoking status, diabetes), social needs questionnaires, and influenza vaccination records were extracted in July 2019 into the REDCap ${ }^{36,37}$ platform. Additional extractions for diagnosis of COPD, frequency of office visits, and HCC-CMS risk scores occurred in February and March 2021. All patients $(N=$ $14,022)$ seen for ambulatory visits in the Department of Family Medicine in a 24-month period from July 2017 to July 2019 were screened for inclusion. Children (age $<18$ years, $N=$ 1098) were excluded from further analysis. A total of 8057 adults had completed the 11-question social needs questionnaire. There were 102 individuals with missing demographic or health data elements, who were excluded from analysis, leaving 7955 analyzed adults. Appendix Table 1 reports characteristics of adults with incomplete social needs screening or missing data elements.

\section{Statistical Analyses}

Bivariable and multivariable logistic regression first modeled current-year and any-year influenza vaccination as a function of the 11 social needs and potential confounder variables, viz., age, sex, race, ethnicity, current smoking status, diabetes, COPD, frequency of primary care office visits with a physician, and CMS-HCC score. Final multivariable models were obtained using stepwise variable selection. Age was treated as a continuous variable. Race was modeled with 4 levels (White, Black/African American, Asian, other/multiple). Because office visits frequency exhibited right skew (e.g., physician visits had a median of 2 , interquartile range [IQR] $1-3$, and range of 0-57 visits), office visit frequency was quantized into three, approximately equally populated levels: $0-1$ office visits per year ( $N=3326), 2-3$ office visits per year $(N=2690)$, or 4 or more office visits per year $(N=1939)$. Similarly, CMS-HCC score was quantized into 3 approximately equally populated levels: low (less than or equal to $0.263, N=2740$ ), moderate (0.263 to $0.691, N=2589$ ), and high (greater than 0.691 , $N=2626$ ). Multicollinearity among social needs and covariates was excluded by calculating variance inflation factors (VIF) for each parameter (Appendix Table 2; maximal VIF 2.37). Sensitivity analyses (Appendix Tables 3-8) evaluated the impact of considering alternative models, viz., (1) collapsing all 11 needs into a single, dichotomous variable (any need, no needs); (2) collapsing the 11 needs into a continuous variable, the number of needs unmet; and (3) controlling for the frequency of primary care office visits including providers of any level (physician, nurse, diabetic educator, etc.) rather than only physicians. We also performed post hoc exploratory subgroup analyses on individuals aged 65 or greater (Appendix Tables 9-11). Association between frequency of office visits with transportation was interrogated using the Wilcoxon rank sum test with continuity correction. Differences in screened vs. un-screened persons were tested using the Wilcoxon rank sum test (age) or chi-squared/Fisher exact tests (all other variables). Statistical analyses were performed using $\mathrm{R}$ version 3.6.1.

\section{Human Subjects Protection}

This study was reviewed by the University of Kansas Medical Center Institutional Review Board and determined to be quality improvement. Data extraction was further approved by the University of Kansas Health System Data Governance committee. Ages 89 years or greater (considered identifiable) were reduced to 88 years to reduce the risk of loss of confidentiality.

\section{RESULTS}

Study population $(N=7955)$ characteristics are summarized in Table 2. The median age was 46 years (IQR: $31-60$ years). Participants were most likely to be female $(62.2 \%)$, nonHispanic (88.9\%), not currently smoking $(85.7 \%)$, and not diabetic $(82.8 \%)$. Most participants were White $(54.6 \%)$, followed by Black (28.8\%).

For current-year vaccination, 3535 (44.4\%) were vaccinated and $4420(55.6 \%)$ were not vaccinated. For any-year vaccination, $5442(68.4 \%)$ were vaccinated and 2513 (31.6\%) were not vaccinated. The prevalence of social needs is shown in Table 1. A total of 1626 (20.4\%) individuals reported at 
Table 2 Population Characteristics

\begin{tabular}{|c|c|}
\hline Characteristic & No. (\%) \\
\hline Age, y, median (IQR) & $46(31-60)$ \\
\hline \multicolumn{2}{|l|}{ Race } \\
\hline Asian & $269(3.4)$ \\
\hline Black/African American & $2288(28.8)$ \\
\hline White & $4343(54.6)$ \\
\hline Other or multiple & $1055(13.3)$ \\
\hline \multicolumn{2}{|l|}{ Sex } \\
\hline Male & $3005(37.8)$ \\
\hline Female & $4950(62.2)$ \\
\hline \multicolumn{2}{|l|}{ Ethnicity } \\
\hline Hispanic, Latin, or Spanish origin & $881(11.1)$ \\
\hline Not of Hispanic, Latin, or Spanish origin & $7074(88.9)$ \\
\hline \multicolumn{2}{|l|}{ Smoking status } \\
\hline Current smoker & $1138(14.3)$ \\
\hline Not a current smoker & $6817(85.7)$ \\
\hline \multicolumn{2}{|l|}{ Diabetes } \\
\hline Present & $1368(17.2)$ \\
\hline Absent & $6587(82.8)$ \\
\hline \multicolumn{2}{|l|}{ Chronic obstructive pulmonary disease } \\
\hline Present & $235(3.0)$ \\
\hline Absent & $7720(97.0)$ \\
\hline \multicolumn{2}{|l|}{ Primary care visits with physician/APP } \\
\hline $0-1$ visits per year & $3326(41.8)$ \\
\hline $2-3$ visits per year & $2690(33.8)$ \\
\hline 4 or more visits per year & $1939(24.4)$ \\
\hline \multicolumn{2}{|l|}{ Primary care visits with any provider level } \\
\hline $0-1$ visits per year & $3102(39.0)$ \\
\hline $2-3$ visits per year & $2659(33.4)$ \\
\hline 4 or more visits per year & $2194(27.6)$ \\
\hline \multicolumn{2}{|l|}{ CMS-HCC risk score } \\
\hline Low & $2740(34.4)$ \\
\hline Moderate & $2589(32.5)$ \\
\hline High & $2626(33.0)$ \\
\hline \multicolumn{2}{|l|}{ Current-year immunization status } \\
\hline Immunized & $3535(44.4)$ \\
\hline Not immunized & $4420(55.6)$ \\
\hline \multicolumn{2}{|l|}{ Any-year immunization status } \\
\hline Immunized & $5442(68.4)$ \\
\hline Not immunized & $2513(31.6)$ \\
\hline
\end{tabular}

$I Q R$, interquartile range; $A P P$, advanced (nursing) practice provider; CMS-HCC, Centers for Medicare and Medicaid Services Hierarchical Condition Categories

least one unfulfilled social need. The most common unfulfilled social needs were transportation $(N=569,7.2 \%)$, companionship $(N=514,6.5 \%)$, and food security $(N=494,6.2 \%)$.

The distribution of predictor variables, stratified by vaccination status, is reported in Table 3. Bivariable and multivariable odds ratios (OR) before variable selection are reported in Table 4 (current-year) and Table 5 (any-year). Table 6 reports final multivariable odds ratios after stepwise variable selection.

Among the social needs, transportation vulnerability and poor health literacy were associated with influenza vaccination. Individuals with transportation vulnerability were less likely to be vaccinated against influenza (current-year aOR $0.65,95 \%$ CI: $0.53-0.78, p<0.001$; any-year aOR $0.58,95 \%$ CI: $0.47-0.71, p<0.001)$. This effect does not appear be solely mediated by frequency of office visits; we failed to find an association between office visit frequency and transportation vulnerability (Wilcoxon $p=0.29$; Appendix Table 12) and inclusion of office visit frequency in the multivariable model does not abolish the significance of transportation vulnerability (Tables 4, 5, and 6). Poor health literacy, interestingly, significantly increased the likelihood of anyyear (but not current-year) influenza vaccination (any-year aOR 1.30, 95\% CI: 1.01-1.69, $p=0.043$ ).

Significant associations were also detected among other covariables (Table 6). Risk factors for lack of current-year and/or any-year influenza vaccination included smoking, Black/African American race, and other/multiple race (current-year only). Factors associated with greater influenza vaccination included age, female sex (any-year only), diabetes, greater CMS-HCC risk score, and greater frequency of primary care visits.

We considered that the choices we made in building the statistical models might have impacted our results. Sensitivity analyses therefore evaluated alternative models (see Appendix Text on Sensitivity Analyses and Appendix Tables 3-8). None of these sensitivity analyses substantially altered our conclusions that transportation vulnerability and poor health literacy are associated with vaccination behavior.

Finally, we performed post hoc exploratory subgroup analysis of the 1339 persons aged 65 years and greater (Appendix Tables 9, 10, 11). Because these are post hoc analyses, they should be considered merely hypothesisgenerating. Factors associated with vaccine uptake in this subgroup included age (any-year only), Black race, diabetes, smoking status, greater CMS-HCC risk score, and greater office visit frequency. Gender was not associated with vaccination in this subgroup. Moreover, neither transportation vulnerability nor health literacy was significantly associated with vaccine uptake. Point estimates for the odds ratio of vaccine uptake generally remain similar in the older population as compared to all adults $(N=7955)$, but the confidence intervals are significantly wider in the subgroup due to the smaller sample size. Interestingly, poor home safety and prescription costs emerge as factors negatively associated with vaccine uptake in the any-year model (home safety aOR $0.45,95 \%$ CI $0.24-0.87, p=0.016$; prescription costs aOR 0.05 , $95 \%$ CI $0.00-0.76, p=0.032$ ). Note, however, that prescription costs were only a problem for $0.2 \%$ of older adults (Appendix Table 11). Future studies-using a larger sample size - should consider probing associations between vaccine uptake in older adults and (1) transportation vulnerability, (2) health literacy, (3) home safety, and/or (4) prescription drug costs.

\section{CONCLUSIONS}

We evaluated sociodemographic factors associated with current-year and any-year influenza vaccine uptake in English-speaking adults seeking primary care at an academic medical center in the Midwestern United States. Consistent with prior analyses, we found that age, $4,6,9-13,15,17,18,25,27,38$ sex, ${ }^{4,17,19}$ race, ${ }^{5,12,16,28}$ chronic illness, ${ }^{6,9,11,13,17,25,27,38}$ and 
Table 3 Predictor Variables Stratified by Current-Year and Any-Year Immunization Status

\begin{tabular}{|c|c|c|c|c|c|}
\hline \multirow[t]{2}{*}{ Variable } & \multirow[t]{2}{*}{ Total } & \multicolumn{2}{|c|}{ Current-year no. (\%) } & \multicolumn{2}{|c|}{ Any-year no. (\%) } \\
\hline & & Imm & Not imm & Imm & Not imm \\
\hline Age, y, median (IQR) & & $52(34-64)$ & $41(30-56)$ & $49(32-62)$ & $41(30-55)$ \\
\hline \multicolumn{6}{|l|}{ Sex } \\
\hline Male & 3005 & $1306(43.5)$ & $1699(56.5)$ & $1951(64.9)$ & $1054(35.1)$ \\
\hline Female & 4950 & $2229(45.0)$ & $2721(55.0)$ & $3491(70.5)$ & $1459(29.5)$ \\
\hline \multicolumn{6}{|l|}{ Race } \\
\hline Asian & 269 & $113(42.0)$ & $156(58.0)$ & $192(71.4)$ & 77 (28.6) \\
\hline Black & 2288 & $892(39.0)$ & $1396(61.0)$ & $1495(65.3)$ & $793(34.7)$ \\
\hline White & 4343 & $2103(48.4)$ & $2240(51.6)$ & $3032(69.8)$ & $1311(30.2)$ \\
\hline Other/multiple & 1055 & $427(40.5)$ & $628(59.5)$ & $723(68.5)$ & $332(31.5)$ \\
\hline \multicolumn{6}{|l|}{ Ethnicity } \\
\hline Hispanic, Latin, or Spanish origin & 881 & $374(42.5)$ & $507(57.5)$ & $621(70.5)$ & $260(29.5)$ \\
\hline Not of Hispanic, Latin, or Spanish origin & 7074 & $3161(44.7)$ & $3913(55.3)$ & $4821(68.2)$ & $2253(31.8)$ \\
\hline \multicolumn{6}{|l|}{ Diabetes } \\
\hline Not diabetic & 6587 & 2738 (41.6) & $3849(58.4)$ & $4318(65.6)$ & $2269(34.4)$ \\
\hline Diabetic & 1368 & $797(58.3)$ & $571(41.7)$ & $1124(82.2)$ & $244(17.8)$ \\
\hline \multicolumn{6}{|l|}{ COPD } \\
\hline Absent & 7720 & $3396(44.0)$ & $4324(56.0)$ & $5256(68.1)$ & $2464(31.9)$ \\
\hline Present & 235 & $139(59.1)$ & $96(40.9)$ & $186(79.1)$ & $49(20.9)$ \\
\hline \multicolumn{6}{|l|}{ Smoking } \\
\hline Not currently & 6817 & $3134(46.0)$ & $3683(54.0)$ & $4764(69.9)$ & $2053(30.1)$ \\
\hline Currently & 1138 & $401(35.2)$ & $737(64.8)$ & $678(59.6)$ & $460(40.4)$ \\
\hline \multicolumn{6}{|l|}{ Childcare } \\
\hline Not at risk & 7827 & $3472(44.4)$ & $4355(55.6)$ & $5352(68.4)$ & 2475 (31.6) \\
\hline At risk & 128 & $63(49.2)$ & $65(50.8)$ & $90(70.3)$ & $38(29.7)$ \\
\hline \multicolumn{6}{|l|}{ Companionship } \\
\hline Not at risk & 7441 & $3289(44.2)$ & $4152(55.8)$ & $5075(68.2)$ & $2366(31.8)$ \\
\hline At risk & 514 & $246(47.9)$ & $268(52.1)$ & $367(71.4)$ & $147(28.6)$ \\
\hline Food security & & & & & \\
\hline Not at risk & 7461 & $3332(44.7)$ & $4129(55.3)$ & $5113(68.5)$ & $2348(31.5)$ \\
\hline At risk & 494 & $203(41.1)$ & $291(58.9)$ & $329(66.6)$ & $165(33.4)$ \\
\hline Health literacy & & & & & \\
\hline Not at risk & 7498 & $3340(44.5)$ & $4158(55.5)$ & $5119(68.3)$ & $2379(31.7)$ \\
\hline At risk & 457 & $195(42.7)$ & $262(57.3)$ & $323(70.7)$ & $134(29.3)$ \\
\hline Home safety & & & & & \\
\hline Not at risk & 7616 & $3381(44.4)$ & $4235(55.6)$ & $5200(68.3)$ & $2416(31.7)$ \\
\hline At risk & 339 & $154(45.4)$ & $185(54.6)$ & $242(71.4)$ & $97(28.6)$ \\
\hline Housing & & & & & \\
\hline Not at risk & 7901 & $3510(44.4)$ & $4391(55.6)$ & $5408(68.4)$ & 2493 (31.6) \\
\hline At risk & 54 & $25(46.3)$ & $29(53.7)$ & $34(63.0)$ & $20(37.0)$ \\
\hline Neighborhood safety & & & & & \\
\hline Not at risk & 7594 & $3378(44.5)$ & $4216(55.5)$ & $5196(68.4)$ & 2398 (31.6) \\
\hline At risk & 361 & $157(43.5)$ & $204(56.5)$ & $246(68.1)$ & $115(31.9)$ \\
\hline Provider health costs & & & & & \\
\hline Not at risk & 7702 & 3434 (44.6) & $4268(55.4)$ & $5275(68.5)$ & $2427(31.5)$ \\
\hline At risk & 253 & $101(39.9)$ & $152(60.1)$ & $167(66.0)$ & $86(34.0)$ \\
\hline Prescription costs & & & & & \\
\hline Not at risk & 7855 & $3498(44.5)$ & $4357(55.5)$ & $5378(68.5)$ & $2477(31.5)$ \\
\hline At risk & 100 & $37(37.0)$ & $63(63.0)$ & $64(64.0)$ & $36(36.0)$ \\
\hline Transportation & & & & & \\
\hline Not at risk & 7386 & $3346(45.3)$ & $4040(54.7)$ & $5108(69.2)$ & $2278(30.8)$ \\
\hline At risk & 569 & $189(33.2)$ & $380(66.8)$ & $334(58.7)$ & $235(41.3)$ \\
\hline Utilities & & & & & \\
\hline Not at risk & 7679 & $3423(44.6)$ & $4256(55.4)$ & $5254(68.4)$ & 2425 (31.6) \\
\hline At risk & 276 & $112(40.6)$ & $164(59.4)$ & $188(68.1)$ & $88(31.9)$ \\
\hline Primary care visits with physician/APP & & & & & \\
\hline $0-1$ visits per year & 3326 & $1002(30.1)$ & $2324(69.9)$ & $1899(57.1)$ & $1427(42.9)$ \\
\hline $2-3$ visits per year & 2690 & $1296(48.2)$ & $1394(51.8)$ & $1950(72.5)$ & $740(27.5)$ \\
\hline 4 or more visits per year & 1939 & $1237(63.8)$ & $702(36.2)$ & $1593(82.2)$ & $346(17.8)$ \\
\hline Primary care visits with any provider level & & & & & \\
\hline $0-1$ visits per year & 3102 & $871(28.1)$ & $2231(71.9)$ & $1716(55.3)$ & $1386(44.7)$ \\
\hline $2-3$ visits per year & 2659 & $1278(48.1)$ & $1381(51.9)$ & $1933(72.7)$ & $726(27.3)$ \\
\hline 4 or more visits per year & 2194 & $1386(63.2)$ & $808(36.8)$ & $1793(81.7)$ & $401(18.3)$ \\
\hline CMS-HCC risk score & & & & & \\
\hline Low & 2740 & $924(33.7)$ & $1816(66.3)$ & 1577 (57.6) & $1163(42.4)$ \\
\hline Moderate & 2589 & 1125 (43.5) & $1464(56.5)$ & $1772(68.4)$ & $817(31.6)$ \\
\hline High & 2626 & $1486(56.6)$ & $1140(43.4)$ & 2093 (79.7) & $533(20.3)$ \\
\hline
\end{tabular}

Imm, immunized; IQR, interquartile range; APP, advanced (nursing) practice provider; CMS-HCC, Centers for Medicare and Medicaid Services Hierarchical Condition Categories 
Table 4 Bivariable and Multivariable Odds Ratios for Current-Year Influenza Vaccination Before Variable Selection

\begin{tabular}{|c|c|c|c|c|c|}
\hline \multirow[t]{2}{*}{ Variable } & \multicolumn{2}{|l|}{ Bivariable (crude) } & \multicolumn{3}{|c|}{ Multivariable (adjusted) } \\
\hline & cOR $(95 \%$ CI $)$ & $p$ value & aOR $(95 \%$ CI) & $p$ value & Sig \\
\hline Age, decades & $1.25(1.22-1.29)$ & $<0.001$ & $1.15(1.11-1.19)$ & $<0.001$ & $* * *$ \\
\hline Female & $1.07(0.97-1.17)$ & 0.172 & $1.06(0.96-1.17)$ & 0.262 & \\
\hline \multicolumn{6}{|l|}{ Race } \\
\hline White & Ref. & & Ref. & & \\
\hline Asian & $0.77(0.60-0.99)$ & 0.041 & $0.84(0.64-1.09)$ & 0.193 & \\
\hline Black & $0.68(0.61-0.75)$ & $<0.001$ & $0.53(0.47-0.60)$ & $<0.001$ & $* * *$ \\
\hline Other/multiple & $0.72(0.63-0.83)$ & $<0.001$ & $0.72(0.58-0.89)$ & 0.002 & $* *$ \\
\hline Non-Hispanic & $1.10(0.95-1.26)$ & 0.209 & $1.08(0.86-1.35)$ & 0.506 & \\
\hline Diabetic & $1.96(1.74-2.21)$ & $<0.001$ & $1.22(1.06-1.40)$ & 0.007 & $* *$ \\
\hline COPD & $1.84(1.42-2.41)$ & $<0.001$ & $0.97(0.73-1.30)$ & 0.858 & \\
\hline Current smoker & $0.64(0.56-0.73)$ & $<0.001$ & $0.60(0.52-0.69)$ & $<0.001$ & $* * *$ \\
\hline \multicolumn{6}{|l|}{ Unmet social need } \\
\hline Childcare & $1.22(0.86-1.73)$ & 0.273 & $1.07(0.71-1.62)$ & 0.752 & \\
\hline Companionship & $1.16(0.97-1.39)$ & 0.107 & $1.07(0.87-1.33)$ & 0.517 & \\
\hline Food insecurity & $0.86(0.72-1.04)$ & 0.123 & $0.99(0.78-1.25)$ & 0.933 & \\
\hline Health literacy & $0.93(0.76-1.12)$ & 0.433 & $1.01(0.79-1.28)$ & 0.950 & \\
\hline Home safety & $1.04(0.84-1.30)$ & 0.708 & $0.96(0.75-1.23)$ & 0.754 & \\
\hline Housing & $1.08(0.63-1.84)$ & 0.783 & $1.09(0.57-2.05)$ & 0.796 & \\
\hline Neighborhood safety & $0.96(0.78-1.19)$ & 0.711 & $1.06(0.81-1.38)$ & 0.669 & \\
\hline Provider health costs & $0.83(0.64-1.06)$ & 0.142 & $0.82(0.60-1.12)$ & 0.217 & \\
\hline Prescription costs & $0.73(0.48-1.09)$ & 0.133 & $0.96(0.61-1.49)$ & 0.850 & \\
\hline Transportation & $0.60(0.50-0.72)$ & $<0.001$ & $0.65(0.52-0.81)$ & $<0.001$ & $* * *$ \\
\hline Utilities & $0.85(0.66-1.08)$ & 0.190 & $0.99(0.75-1.31)$ & 0.953 & \\
\hline \multicolumn{6}{|l|}{ CMS-HCC risk score } \\
\hline Low & Ref. & & Ref. & & \\
\hline Moderate & $1.51(1.35-1.69)$ & $<0.001$ & $1.09(0.96-1.24)$ & 0.198 & \\
\hline High & $2.56(2.29-2.86)$ & $<0.001$ & $1.39(1.19-1.62)$ & $<0.001$ & $* * *$ \\
\hline \multicolumn{6}{|l|}{ Primary care office visits } \\
\hline $0-1$ per year & Ref. & & Ref. & & \\
\hline 2-3 per year & $2.16(1.94-2.40)$ & $<0.001$ & $2.00(1.79-2.23)$ & $<0.001$ & $* * *$ \\
\hline 4 or more per year & $4.09(3.63-4.60)$ & $<0.001$ & $3.63(3.19-4.14)$ & $<0.001$ & $* * *$ \\
\hline
\end{tabular}

cOR, crude (bivariable) odds ratio; CI, confidence interval; aOR, adjusted (multivariable) odds ratio; Sig, significance relative to the multivariable model $(* p<0.05, * * p<0.01, * * * p<0.001) ;$ Ref., Reference; CMS-HCC, Centers for Medicare and Medicaid Services Hierarchical Condition Categories

Table 5 Bivariable and Multivariable Odds Ratios for Any-Year Influenza Vaccination Before Variable Selection

\begin{tabular}{|c|c|c|c|c|c|}
\hline \multirow[t]{2}{*}{ Variable } & \multicolumn{2}{|l|}{ Bivariable (crude) } & \multicolumn{3}{|c|}{ Multivariable (adjusted) } \\
\hline & cOR $(95 \%$ CI) & $p$ value & aOR (95\% CI) & $p$ value & Sig \\
\hline Age, decades & $1.19(1.16-1.23)$ & $<0.001$ & $1.05(1.01-1.09)$ & 0.008 & $* *$ \\
\hline Female & $1.29(1.17-1.42)$ & $<0.001$ & $1.26(1.14-1.40)$ & $<0.001$ & $* * *$ \\
\hline \multicolumn{6}{|l|}{ Race } \\
\hline White & Ref. & & Ref. & & \\
\hline Asian & $1.08(0.82-1.42)$ & 0.588 & $1.21(0.92-1.61)$ & 0.187 & \\
\hline Black & $0.82(0.73-0.91)$ & $<0.001$ & $0.65(0.58-0.74)$ & $<0.001$ & $* * *$ \\
\hline Other/multiple & $0.94(0.81-1.09)$ & 0.417 & $0.87(0.71-1.08)$ & 0.216 & \\
\hline Non-Hispanic & $0.90(0.77-1.04)$ & 0.160 & $0.94(0.75-1.19)$ & 0.614 & \\
\hline Diabetic & $2.42(2.09-2.81)$ & $<0.001$ & $1.46(1.24-1.73)$ & $<0.001$ & $* * *$ \\
\hline COPD & $1.78(1.31-2.47)$ & $<0.001$ & $1.00(0.72-1.43)$ & 0.981 & \\
\hline Current smoker & $0.64(0.56-0.72)$ & $<0.001$ & $0.61(0.53-0.70)$ & $<0.001$ & $* * *$ \\
\hline \multicolumn{6}{|l|}{ Unmet social need } \\
\hline Childcare & $1.10(0.75-1.62)$ & 0.641 & $0.96(0.62-1.52)$ & 0.856 & \\
\hline Companionship & $1.16(0.96-1.42)$ & 0.132 & $1.05(0.83-1.33)$ & 0.701 & \\
\hline Food insecurity & $0.92(0.76-1.11)$ & 0.372 & $0.99(0.77-1.26)$ & 0.916 & \\
\hline Health literacy & $1.12(0.91-1.38)$ & 0.283 & $1.30(1.01-1.70)$ & 0.048 & $*$ \\
\hline Home safety & $1.16(0.91-1.48)$ & 0.229 & $1.08(0.83-1.42)$ & 0.556 & \\
\hline Housing & $0.78(0.45-1.39)$ & 0.389 & $0.72(0.38-1.39)$ & 0.325 & \\
\hline Neighborhood safety & $0.99(0.79-1.24)$ & 0.911 & $1.02(0.78-1.35)$ & 0.868 & \\
\hline Provider health costs & $0.89(0.69-1.17)$ & 0.404 & $0.90(0.65-1.26)$ & 0.545 & \\
\hline Prescription costs & $0.82(0.55-1.25)$ & 0.341 & $0.93(0.60-1.47)$ & 0.767 & \\
\hline Transportation & $0.63(0.53-0.75)$ & $<0.001$ & $0.58(0.46-0.72)$ & $<0.001$ & $* * *$ \\
\hline Utilities & $0.99(0.76-1.28)$ & 0.915 & $1.08(0.81-1.46)$ & 0.598 & \\
\hline \multicolumn{6}{|l|}{ CMS-HCC risk score } \\
\hline Low & Ref. & & Ref. & & \\
\hline Moderate & $1.60(1.43-1.79)$ & $<0.001$ & $1.36(1.20-1.55)$ & $<0.001$ & $* * *$ \\
\hline High & $2.90(2.57-3.27)$ & $<0.001$ & $1.93(1.64-2.28)$ & $<0.001$ & $* * *$ \\
\hline
\end{tabular}


Table 5. (continued)

\begin{tabular}{|c|c|c|c|c|c|}
\hline \multirow[t]{2}{*}{ Variable } & \multicolumn{2}{|c|}{ Bivariable (crude) } & \multicolumn{3}{|c|}{ Multivariable (adjusted) } \\
\hline & cOR $(95 \% \mathrm{CI})$ & $p$ value & aOR $(95 \%$ CI) & $p$ value & Sig \\
\hline \multicolumn{6}{|l|}{ Primary care office visits } \\
\hline 2-3 per year & $1.98(1.78-2.21)$ & $<0.001$ & $1.75(1.57-1.96)$ & $<0.001$ & $* * *$ \\
\hline 4 or more per year & $3.46(3.03-3.96)$ & $<0.001$ & $2.66(2.30-3.08)$ & $<0.001$ & $* * *$ \\
\hline
\end{tabular}

cOR, crude (bivariable) odds ratio; CI, confidence interval; aOR, adjusted (multivariable) odds ratio; Sig, significance relative to the multivariable model $(* p<0.05, * * p<0.01, * * * p<0.001)$; Ref., Reference; CMS-HCC, Centers for Medicare and Medicaid Services Hierarchical Condition Categories

smoking status $6,10,11,17$ are associated with influenza vaccination.

We identified two social needs that are associated with influenza vaccination uptake: transportation vulnerability, which is associated with decreased immunization (currentyear aOR 0.65 , any-year aOR 0.58 ); and poor health literacy, which - interestingly - is associated with increased any-year immunization (aOR 1.30). Validation of transportation vulnerability as a risk factor in adults is concordant with similar findings in children ${ }^{12}$ and the elderly. ${ }^{8}$ Health literacy has been noted to influence influenza vaccination in older adults, but was noted to be associated with decreased vaccination - not increased vaccination - in a prior analysis of 2668 adults aged 65 years or greater. ${ }^{39}$ We speculate that individuals with low health literacy may simply accept a physician recommendation for vaccination, resulting in higher influenza vaccination rates; future studies may fruitfully interrogate this hypothesis.

The prevalence of transportation vulnerability in our population $(7.2 \%)$ is similar to other reports in primary care or community populations (range $4-12 \%)^{40-43}$ The rate of self- reported poor health literacy in our population $(5.7 \%)$ is far lower than the National Assessment of Adult Literacy (NAAL) estimate of $36 \%{ }^{44}$ There are several possible explanations. The NAAL estimate involves robust analysis of abilities and performance on specific health tasks. Our population is asked a single question related to health literacy and is selfreported, which may contribute to the lower observed rates. Additionally, we included only English speakers which may have higher average health literacy. Our data predict that communities with higher rates of transportation vulnerability, or greater health literacy, may have reduced vaccination uptake; future studies could investigate this hypothesis in communities with different prevalence of social needs.

We further noted diabetes and greater CMS-HCC risk score are associated with increased vaccination, which is in agreement with prior analyses demonstrating increased influenza vaccination uptake in individuals with chronic illnesses, including diabetes. ${ }^{6,9,14,17,25,38}$ Moreover, in our analysis, smoking was associated with decreased influenza vaccination. There is disagreement in the literature over the role of smoking on influenza vaccination: some analyses have reported current

Table 6 Final Multivariable Odds Ratios for Current-Year and Any-Year Vaccination After Stepwise Variable Selection

\begin{tabular}{|c|c|c|c|c|c|c|}
\hline \multirow[t]{2}{*}{ Variable } & \multicolumn{2}{|l|}{ Current-year } & \multirow[b]{2}{*}{ Sig } & \multicolumn{3}{|l|}{ Any-year } \\
\hline & aOR $(95 \% \mathrm{CI})$ & $p$ value & & aOR $(95 \% \mathrm{CI})$ & $p$ value & Sig \\
\hline Age, decades & $1.15(1.11-1.19)$ & $<0.001$ & $* * *$ & $1.05(1.01-1.09)$ & 0.007 & $* *$ \\
\hline Female & - & - & - & $1.26(1.14-1.40)$ & $<0.001$ & $* * *$ \\
\hline \multicolumn{7}{|l|}{ Race } \\
\hline White & Ref. & & & Ref. & & \\
\hline Asian & $0.84(0.65-1.10)$ & 0.205 & & $1.21(0.91-1.61)$ & 0.195 & \\
\hline Black & $0.53(0.48-0.60)$ & $<0.001$ & $* * *$ & $0.65(0.58-0.74)$ & $<0.001$ & $* * *$ \\
\hline Other/multiple & $0.69(0.59-0.79)$ & $<0.001$ & $* * *$ & $0.91(0.78-1.06)$ & 0.226 & \\
\hline Diabetic & $1.21(1.05-1.39)$ & 0.008 & $* *$ & $1.46(1.24-1.73)$ & $<0.001$ & $* * *$ \\
\hline Current smoker & $0.59(0.51-0.68)$ & $<0.001$ & $* * *$ & $0.60(0.53-0.70)$ & $<0.001$ & $* * *$ \\
\hline \multicolumn{7}{|l|}{ Unfulfilled social need } \\
\hline Health literacy & - & - & - & $1.30(1.01-1.69)$ & 0.043 & $*$ \\
\hline Transportation & $0.65(0.53-0.78)$ & $<0.001$ & $* * *$ & $0.58(0.47-0.71)$ & $<0.001$ & $* * *$ \\
\hline \multicolumn{7}{|l|}{ CMS-HCC risk score } \\
\hline Low & Ref. & & & Ref. & & \\
\hline Moderate & $1.09(0.96-1.24)$ & 0.185 & & $1.36(1.20-1.55)$ & $<0.001$ & $* * *$ \\
\hline High & $1.40(1.20-1.62)$ & $<0.001$ & $* * *$ & $1.94(1.65-2.28)$ & $<0.001$ & $* * *$ \\
\hline \multicolumn{7}{|l|}{ Primary care physician visits } \\
\hline $0-1$ visits per year & Ref. & & & Ref. & & \\
\hline $2-3$ visits per year & $2.01(1.80-2.24)$ & $<0.001$ & $* * *$ & $1.75(1.56-1.96)$ & $<0.001$ & $* * *$ \\
\hline 4 or more visits per year & $3.63(3.20-4.13)$ & $<0.001$ & $* * *$ & $2.66(2.30-3.07)$ & $<0.001$ & $* * *$ \\
\hline
\end{tabular}

aOR, adjusted (multivariable) odds ratio; CI, confidence interval; Sig, significance $\left({ }^{*} p<0.05, * * p<0.01\right.$, ***p $\left.<0.001\right)$; Ref., Reference; CMS-HCC, Centers for Medicare and Medicaid Services Hierarchical Condition Categories 
smoking as increasing influenza vaccination, ${ }^{6}$ while others report decreased vaccination uptake among smokers. ${ }^{10}$

This study has several limitations. First, data were collected at a single, tertiary care, urban, midwestern, academic medical center. Caution should be exercised when extending these results to community-based clinics and in sub-urban or rural areas. Second, social needs responses are self-reported. The rate of unfulfilled social needs may therefore be underreported due to the perceived stigma of acknowledging a need for assistance. Third, any screening instrument evaluating social needs can simultaneously satisfy only two of the following three criteria: brief, multidomain, or detailed. By selecting a brief (11-item), multidomain screening instrument, each social needs domain was afforded only one question. Multiple related concepts may be thus captured by each question; for example, lack of companionship may reflect either social isolation or loneliness (or both), which are not separated by this analysis. Fourth, several of the social needs are related to insufficient financial resources, but we were unable to obtain more direct markers (e.g., household income) of financial well-being because this information is not captured by the EMR. Fifth, the study was observational and non-randomized; the interaction (if any) between influenza vaccination status and willingness to answer questions about social needs is, formally, not known. Sixth, social needs may fluctuate over time, but our analysis captures only the most recent snapshot. Seventh, influenza vaccination status is partially self-reported. Although there are numerous opportunities for immunization data to be captured in the EMR (see "METHODS"), it is possible that influenza vaccination may have been obtained outside the health system but not reported into the EMR. However, estimates of the total current-year vaccination in our analysis $(44.5 \%)$ is similar to CDC estimates of adult influenza vaccination rate $(46.5 \%)$ in Kansas (the study location) during the 2018-2019 influenza season. ${ }^{45}$ The magnitude of influenza immunization under-reporting in our sample is, therefore, likely to be small.

In summary, transportation vulnerability, smoking status, Black/African American race, and other/multiple race are associated with lack of current-year and/or any-year influenza vaccination, while age, female sex, diabetes, poor health literacy, greater frequency of primary care visits, and greater CMS-HCC risk score are associated with increased vaccination. These data add to accumulating evidence of social and racial disparities in prevention of communicable disease. In view of ongoing efforts to deploy vaccines for the current coronavirus disease 2019 (COVID-19) pandemic, ${ }^{46}$ it is conceivable that persons with risk factors for lack of influenza immunizations may also be at risk for lack of immunization against COVID-19. Efforts to rapidly deploy a novel vaccine may need to specifically plan for outreach to persons experiencing transportation vulnerability, in addition to minority racial groups. Future studies may analyze the effect on influenza vaccination of targeted interventions to influence modifiable risk factors, viz., to (a) alleviate transportation vulnerability and (b) encourage smoking cessation. Moreover, the impact on influenza vaccination status of efforts to reduce sex and racial health disparities should be further assessed.

Supplementary Information The online version contains supplementary material available at https://doi.org/10.1007/s11606-02106902-6.

Contributors: We thank Jovita Ramjisingh for assistance in reviewing the social determinants of health literature. We thank Dr. Naiman Nazir for helpful comments on the manuscript.

Corresponding Author: Daniel J. Parente, MD, PhD; Department of Family Medicine and Community Health, University of Kansas Medical Center, Kansas City, KS, USA (e-mail: dparente@kumc.edu).

Funders This work was not funded, except that it utilized the REDCap platform for data management, which was supported by Clinical and Translational Science Award (CTSA) \#UL1TROO2366 from the National Center for Advancing Translational Sciences (NCATS) awarded to the University of Kansas for Frontiers: University of Kansas Clinical and Translational Science Institute.

\section{Declarations:}

Conflict of Interest: The authors declare that they do not have a conflict of interest.

Disclaimer: The funding source was not involved in the design, conduct, or reporting of this study. The contents are solely the responsibility of the authors and do not necessarily represent the official views of the National Institutes of Health (NIH) or NCATS.

\section{REFERENCES}

1. Grohskopf LA, Alyanak E, Broder KR, Walter EB, Fry AM, Jernigan DB. Prevention and Control of Seasonal Influenza with Vaccines: Recommendations of the Advisory Committee on Immunization Practices - United States, 2019-20 Influenza Season. MMWR Recomm Rep 2019;68(3): 1-21.

2. Rolfes MA, Foppa IM, Garg S, et al. Annual estimates of the burden of seasonal influenza in the United States: a tool for strengthening influenza surveillance and preparedness. Influenza Other Respir Viruses 2018;12(1): 132-7.

3. Centers for Disease Control and Prevention. Flu Vaccination Coverage, United States, 2018-19 Influenza Season. 2019. Available at: https:// www.cdc.gov/flu/fluvaxview/coverage-1819estimates.htm. Accessed 26 Sept 2019

4. Nagata JM, Hernandez-Ramos I, Kurup AS, Albrecht D, VivasTorrealba C, Franco-Paredes C. Social determinants of health and seasonal influenza vaccination in adults $>/=65$ years: a systematic review of qualitative and quantitative data. BMC Public Health 2013;13:388.

5. Crouse Guinn S, Jamison AM, Freimuth VS, An J, Hancock GR. Determinants of influenza vaccination among high-risk Black and White adults. Vaccine. 2017;35(51):7154-9.

6. Barbadoro P, Marigliano A, Di Tondo E, et al. Determinants of influenza vaccination uptake among Italian healthcare workers. Hum Vaccin Immunother 2013;9(4):911-6.

7. Bryant WK, Ompad DC, Sisco S, et al. Determinants of influenza vaccination in hard-to-reach urban populations. Prev Med 2006;43(1):60-70.

8. Burns VE, Ring C, Carroll D. Factors influencing influenza vaccination uptake in an elderly, community-based sample. Vaccine. 2005;23(27):3604-8.

9. Chiatti C, Barbadoro $\mathbf{P}$, Lamura G, et al. Influenza vaccine uptake among community-dwelling Italian elderly: results from a large crosssectional study. BMC Public Health 2011;11:207. 
10. Chiatti C, Barbadoro P, Marigliano A, Ricciardi A, Di Stanislao F, Prospero E. Determinants of influenza vaccination among the adult and older Italian population with chronic obstructive pulmonary disease: a secondary analysis of the multipurpose ISTAT survey on health and health care use. Hum Vaccin 2011;7(10):1021-5.

11. de Andres AL, Garrido PC, Hernandez-Barrera V, Del Pozo Sv, de Miguel AG, Jimenez-Garcia R. Influenza vaccination among the elderly Spanish population: trend from 1993 to 2003 and vaccination-related factors. Eur J Pub Health 2007; 17(3):272-7.

12. Falagas ME, Zarkadoulia E. Factors associated with suboptimal compliance to vaccinations in children in developed countries: a systematic review. Curr Med Res Opin 2008;24(6):1719-41.

13. Landi F, Onder G, Carpenter I, Garms-Homolova V, Bernabei R Prevalence and predictors of influenza vaccination among frail community-living elderly patients: an international observational study. Vaccine. 2005;23(30):3896-901

14. Mangtani P, Breeze E, Kovats S, Ng ES, Roberts JA, Fletcher A Inequalities in influenza vaccine uptake among people aged over 74 years in Britain. Prev Med 2005;41(2):545-53.

15. Pena-Rey I, Perez-Farinos N, Sarria-Santamera A. Factors associated with influenza vaccination among elderly Spanish women. Public Health 2004;118(8):582-7.

16. Rangel MC, Shoenbach VJ, Weigle KA, Hogan VK, Strauss RP, Bangdiwala SI. Racial and ethnic disparities in influenza vaccination among elderly adults. J Gen Intern Med 2005;20(5):426-31.

17. Sarria-Santamera A, Timoner J. Influenza vaccination in old adults in Spain. Eur J Pub Health 2003;13(2):133-7.

18. Schmitz H, Wubker A. What determines influenza vaccination take-up of elderly Europeans? Health Econ 2011;20(11):1281-97.

19. Shemesh AA, Rasooly I, Horowitz P, et al. Health behaviors and their determinants in multiethnic, active Israeli seniors. Arch Gerontol Geriatr 2008;47(1):63-77.

20. Wershof Schwartz A, Clarfield AM, Doucette JT, et al. Disparities in pneumococcal and influenza immunization among older adults in Israel: a cross-sectional analysis of socio-demographic barriers to vaccination. Prev Med 2013;56(5):337-40.

21. de Souto Barreto P, Lapeyre-Mestre M, Vellas B, Rolland Y. Indicators of influenza and pneumococcal vaccination in French nursing home residents in 2011. Vaccine. 2014;32(7):846-51.

22. Nexoe J, Kragstrup J, Sogaard J. Decision on influenza vaccination among the elderly. A questionnaire study based on the Health Belie Model and the Multidimensional Locus of Control Theory. Scand J Prim Health Care 1999; 17(2):105-10

23. Glatman-Freedman A, Nichols $\mathbf{K}$. The effect of social determinants on immunization programs. Hum Vaccin Immunother 2012;8(3):293-301.

24. Looijmans-van den Akker I, van Delden JJ, Verheij TJ, et al. Which determinants should be targeted to increase influenza vaccination uptake among health care workers in nursing homes? Vaccine. 2009;27(34):4724-30

25. Jimenez R, Larrauri A, Carrasco P, Esteban J, Gomez-Lopez LI, Gil A Influenza coverages in Spain and vaccination-related factors in the subgroup aged 50-64 years. Vaccine. 2003;21(25-26):3550-5.

26. Jimenez-Garcia R, Hernandez-Barrera V, Carrasco-Garrido $\mathbf{P}$, de Andres AL, Esteban Pena MM, de Miguel AG. Coverage and predictors of influenza vaccination among adults living in a large metropolitan area in Spain: a comparison between the immigrant and indigenous populations. Vaccine. 2008;26(33):4218-23.

27. Jimenez-Garcia R, Rodriguez-Rieiro C, Hernandez-Barrera V, et al. Negative trends from $2008 / 9$ to $2011 / 12$ seasons in influenza vaccination coverages among high risk subjects and health care workers in Spain. Vaccine. 2014;32(3):350-4.

28. Fiscella K, Franks P, Doescher MP, Saver BG. Disparities in health care by race, ethnicity, and language among the insured: findings from a national sample. Med Care 2002;40(1):52-9.

29. Kroneman MW, van Essen GA. Variations in influenza vaccination coverage among the high-risk population in Sweden in 2003/4 and 2004/5: a population survey. BMC Public Health 2007;7:113.
30. Figueroa JF, Frakt AB, Jha AK. Addressing social determinants of health: time for a polysocial risk score. Jama. 2020;323(16):1553-4.

31. Health Leads. The Health Leads Screening Toolkit. Available at: https:// healthleadsusa.org/resources/the-health-leads-screening-toolkit/. Accessed May 202020.

32. Yue D, Pourat $\mathbf{N}$, Chen $\mathbf{X}$, et al. Enabling services improve access to care, preventive services, and satisfaction among health center patients. Health Aff (Millwood) 2019;38(9): 1468-74.

33. Earls FJ, Brooks-Gunn J, Raudenbush SW, Sampson RJ. Project on Human Development in Chicago Neighborhoods (PHDCN): exposure to violence (subject), wave 1, 1994-1997. Inter-university Consortium for Political and Social Research [distributor]; 2007. https://doi.org/10. 3886/ICPSR13589.v2

34. Pope GC, Kautter J, Ellis RP, et al. Risk adjustment of Medicare capitation payments using the CMS-HCC model. Health Care Financ Rev 2004;25(4):119-41.

35. Yeatts JP, Sangvai D. HCC coding, risk adjustment, and physician income: what you need to know. Fam Pract Manag 2016;23(5):24-7.

36. Harris PA, Taylor R, Minor BL, et al. The REDCap consortium: building an international community of software platform partners. J Biomed Inform. 2019;95:103208.

37. Harris PA, Taylor R, Thielke R, Payne J, Gonzalez N, Conde JG. Research electronic data capture (REDCap)-a metadata-driven methodology and workflow process for providing translational research informatics support. J Biomed Inform 2009;42(2):377-81

38. Jimenez-Garcia R, Esteban-Vasallo MD, Rodriguez-Rieiro C, et al. Coverage and predictors of vaccination against 2012/13 seasonal influenza in Madrid, Spain: analysis of population-based computerized immunization registries and clinical records. Hum Vaccin Immunother 2014;10(2):449-55.

39. Bennett IM, Chen J, Soroui JS, White S. The contribution of health literacy to disparities in self-rated health status and preventive health behaviors in older adults. Ann Fam Med 2009;7(3):204-11.

40. Sundar KR. Universal screening for social needs in a primary care clinic: a quality improvement approach using the Your Current Life Situation Survey. Perm J 2018;22:18-089.

41. Page-Reeves J, Kaufman W, Bleecker M, et al. Addressing socia determinants of health in a clinic setting: the WellRx Pilot in Albuquerque, New Mexico. J Am Board Fam Med 2016;29(3):414-8.

42. Lewis CC, Wellman R, Jones SMW, et al. Comparing the performance of two social risk screening tools in a vulnerable subpopulation. J Family Med Prim Care 2020;9(9):5026-34.

43. Meyer D, Lerner E, Phillips A, Zumwalt K. Universal screening of social determinants of health at a large US academic medical center, 2018. Am J Public Health 2020;110(S2):S219-s21.

44. Kutner M, Greenburg E, Jin Y, Paulsen C. The health literacy of America's adults: results from the 2003 National Assessment of Adult Literacy. In: U.S. Department of Education NCfES, ed; 2016.

45. Centers for Disease Control and Prevention. 2018-19 Influenza Season Vaccination Coverage Dashboard. 2019. Available at: https://www.cdc gov/flu/fluvaxview/reportshtml/reporti1819/reportii/index.html. Accessed 13 May 2020.

46. Collins FS, Stoffels P. Accelerating COVID-19 Therapeutic Interventions and Vaccines (ACTIV): an unprecedented partnership for unprecedented times. JAMA 2020;323(24):2455-7.

Publisher's Note: Springer Nature remains neutral with regard to jurisdictional claims in published maps and institutional affiliations. 